\title{
Prevention of Cerebral Palsy and Fetal Demise with Hypoxia Index, FHR Score and Pathologic Sinusoidal FHR in Fetal Monitoring
}

\author{
Kazuo Maeda ${ }^{1 *}$, Masaji Utsu \\ ${ }^{1}$ Honorary professor of Obstetrics and Gynecology, Tottori University Medical School, Yonago, Japan \\ ${ }^{2}$ Department of Obstetrics and Gynecology, Seirei Mikatahara hospital., Hamamatsu, Japan
}

Correspondence to: Kazuo Maeda, Honorary professor of Obstetrics and Gynecology, Tottori University Medical School, Yonago, 3-125 Nadamachi, Yonago-shi, Tottoriken, 683-0835, Japan; E-mail: maefak@mocha.ocn.ne.jp

Received date: September 22, 2020; Accepted date: October 01, 2020; Published date: October 06, 2020

Citation: Maeda K, Utsu M (2020) Prevention of Cerebral Palsy and Fetal Demise with Hypoxia Index, FHR Score and Pathologic Sinusoidal FHR in Fetal Monitoring. J Obst Gynecol Surg 1(2): pp. 1-3.

Copyright: (C2020 Maeda K, et al. This is an open-access article distributed under the terms of the Creative Commons Attribution License, which permits unrestricted use, distribution and reproduction in any medium, provided the original author and source are credited.

\section{ABSTRACT}

Although fetal deaths was decreased by intrapartum Fetal Heart Rate (FHR) monitoring, infantile cerebral palsy was not decreased in Dublin trials of Electric Fetal Monitor (EFM), thus, an analysis to reduce cerebral palsy was studied, where cerebral palsy is prevented by setting the threshold of hypoxia index at 24 or less, in the analysis of FHR deceleration.

\section{Keywords:}

Fetal heart rate monitoring, Late deceleration, Hypoxia Index, Cerebral palsy, Numeric threshold

\section{Introduction}

Electronic Fetal Monitors (EFM) were internal method using fetal scalp ECG electrode and intrauterine pressure tracing, where Fetal Heart Rate (FHR) monitoring started limitedly after the rupture of the membrane, thus external monitor with fetal heart sound was used by Hammacher and Maeda, and now mainly by ultrasonic Doppler autocorrelation fetal heart rate meter is distributed in the world and the uterine contraction by tocodynamometer.

Although hypoxic FHR decelerations (transient bradycardia) classified into early, late, and variable decelerations with visual observations, where late deceleration of regular FHR change was ominous [1], while the loss of baseline variability was ominousin Hon and Hammaher's FHR diagnosis [1,2], while it was not reported to be inevitable fetal brain damage followed by cerebral palsy [3]. The separation of pathologic and physiologic sinusoidal FHR was unable by CTG, while it was possible by actocadiogram [4] with the regular fetal movements. The FHR pattern classification was vague in fetal outcome prediction on several occasions, e.g. a variable deceleration was "variable and early deceleration" by Dr. Hon. Thus, numeric objective analysis is required in the intrapartum fetal monitoring, including the artificial neural network analysis [5] or FHR score [6] in the update trend to introduce quantitative FHR analysis.

\section{Methods}

\section{Stable FHR}

The FHR of resting fetus is an example of stable FHR, which is composed of around $120 \mathrm{bpm}$ heart rate, normally overlapped by small irregular FHR variability, of which amplitude is $2-5 \mathrm{bpm}$. There is transient tachycardia, which is FHR rise more than 20 bpm for $5 \mathrm{~min}$, which was supposed to be fetal intraputerine cry. Mild continuous FHR rise is found in continuous fetal respiration and fetal hiccupping motion. Fetal respiratory movement is 1 cps spikes. Continuous 2-sec interval spikes are fetal hiccupping movements which also continuous $3 \mathrm{bpm}$ FHR spikes, but not accompany FHR acceleration due to the absence of fetal movement burst.

\section{FHR decrease}

Common FHR patterns were early, late, mild, and severe variable decelerations, in fetal outcome discussion in visual analysis, where no numeric criteria was shown even in the delay time of late deceleration [1], which was shown to be 20 sec by Check et al. later. Thus, the diagnosis with FHR pattern classifications was controversial even in the memory of the author. Thus, the author firstly created an objective FHR score in 1969 [6], which predicted Apgar score and $\mathrm{UApH}$ [5], then fetal outcome diagnosis with artificial neural network analysis [5]. The author created Actocardiogram, which analyses fetal heart rate with fetal movement, and clarified the relationship between fetal heart rate and movement [7], where triangular FHR acceleration is originated in fetal motion created in the midbrain [8], which changed square shape fetal movement burst to triangular FHR with the integral function of midbrain with 7-sec delay. The simulation of an adult also developed a triangular heart rate from square shape leg movements [7], Thus, it was clear that FHR increases by the fetal brain excited by fetal movements. The function clarified the developing mechanism of physiologic sinusoidal fetal heart rate, where the physiologic sinusoidal is the synchronization of fetal brain to fetal cyclic mouthing or respiratory movements, which separatsphysiologic sinusoidal FHR from pathologic sinusoidal one, which accompanied no fetal movement $[9,10]$.

As fetal brain function to rise heart rate is suppressed by the hypoxia, where firstly FHR acceleration is lost in "non-reactive heart rate", while FHR baseline variability is present in mild hypoxia, thus, the baseline variability was the final response of the fetal brain to minor fetal movements, then the variability is lost in the most severe irreversible hypoxic fetal brain damage. Thus, early cesarean delivery is performed with the purpose to prevent fetal damage before the loss of FHR baseline variability, thus, the delivery after the loss of variability cures fetal life but unable to prevent cerebral palsy due to incurable fetal brain damage in severe hypoxia [11], while the threshold was unknown in the past, but it will be discussed in the present report. 


\section{FHR fall}

FHR falls in fetal hypoxia, where the vagal nerve center located in medulla oblongata is excited by the hypoxic stimulation to reduce FHR, then develop fetal bradycardia in long hypoxia, thus, FHR deceleration develops in transient hypoxia, namely, hypoxic fetal bradycardia is a vagal nerve excitation, but not immediate fetal damage, namely, frequently repeated decelerations or prolonged fetal bradycardia develops fetal brain damage, which is shown by hypoxia index as follows.

\section{Threshold of Hypoxia Index to damage fetal brain}

It was mandatoryto set quantitative numericthreshold to prevent fetal brain damage, which was shown by the loss of baseline variability, while there was no threshold in fetal monitoring in the past, thus, the author tried to study late FHR deceleration, looking for its hypoxic effect. The author experienced a case of 3 connected typical late decelerations with $45 \mathrm{sec}$ lag time, who born fully normal neonate after cesarean delivery, where the Apgar score was 9, while a case of late decelerations repeated for 50 minutes due to refusal of cesarean delivery developed severe neonatal asphyxia of which Apgar score was 3 , associated severe brain damage, which ended by the death in the brain hemorrhage, namely, fetal typically characteristic late deceleration repeated 3 times dose not develop fetal damage, while frequently repeated decelerations 50 minutes developed Apgar3 brain-damaged neonate. Also, there was another definition, that a late deceleration was diagnosed limitedly after 15 or more minutes repetition of the decelerations, Thus, it is confirmed that frequent repetition of decelerations is effective to develophypoxic damage, due to frequent hypoxic damages to the fetus, but not by the characteristic late deceleration pattern, Thus, the author decided to clinically confirm the threshold level, which was defined by the repetition of decelerations.

Namely, the novel Hypoxia Index=The sum of all deceleration durations ( $\mathrm{min}$ ) in the full course of fetal monitoring, divided by the lowest FHR (bpm), which indicates the intensity of hypoxia, then multiplied by 100 to keep the index to be an integer (Figure 1). It was believed that the fetal brain-damaging threshold exists between 3 times decelerations and 50 minutes repetition of decelerations.

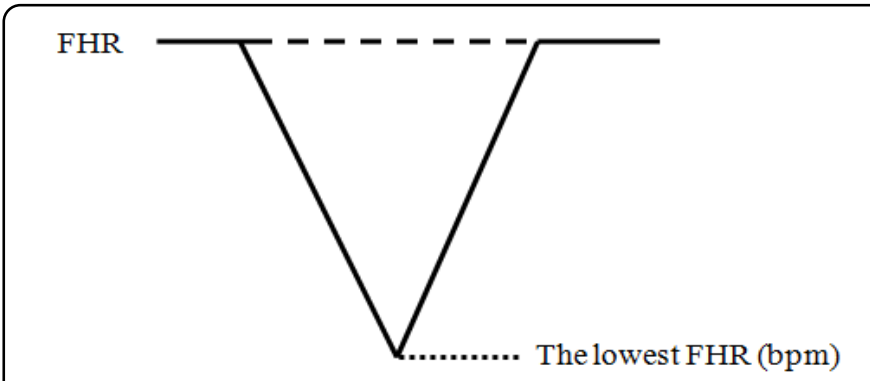

Figure 1: Deceleration duration (Min).

We collected retrospectively 22 cases of infants who were diagnosed by a pediatric clinic, and their intrapartum FHR records were found in the obstetric ward, where 6 cases were cerebral palsy and 16 cases were not cerebral palsy. The hypoxia index of the two groups was compared (Table 1).

Table 1: The frequency of cerebral palsy was $6 / 6(100 \%)$ in 6 cases of 25 or more of hypoxia index, while it was $0 / 16(0 \%)$ in 16 cases whose hypoxia index was 24 or less. There was a significant difference in the number of cerebral palsy in the two groups $(p=0.000008<0.05)$.

\begin{tabular}{|l|l|l|l|}
\hline Hypoxia Index & Total No. & No. of Cerebral palsy & Frequency of Cerebral palsy \\
\hline 25 or more & 6 & $0(0 \%)$ & $6(100 \%)$ \\
\hline 24 or less & 16 & $16(100 \%)$ & $0(0 \%)$ \\
\hline
\end{tabular}

\section{Results and Discussion}

The hypoxia index $(\mathrm{HI})$ of all 6 cerebral palsy cases was 25 or more, but 0 cerebral palsies in 24 or less $\mathrm{HI}$, while the index of all 16 no cerebral palsy cases was 24 or less but 0 in 25 or more HI. The numeric threshold existed between 24 and 25 of the hypoxia index (Table 1). The chi-square test $p$ was 0.000008 , almost zero, and there was a significant difference between the HI 25 or more and 24 or less.

As there was no cerebral palsy in 16 cases, whose hypoxia index was 24 or less, and diagnostic error was almost zero, it would true that cerebral palsy is prevented, if the $\mathrm{HI}$ is kept at the level of 24 or less in fetal monitoring.

The cases whose hypoxia index is $\mathbf{2 5}$ or more can receive early treatment of cerebral palsy even in the neonatal period, as it is possible to develop cerebral palsy if the index is 25 or more. Early treatment may improve the therapeutic effect.

As late deceleration develops to stop placental maternal blood flow by the compression of the pelvic artery by contracted pregnant uterus in the supine posture, and late deceleration disappeared by changing maternal posture to lateral one from supine $[11,12]$, the author recommends changing maternal posture to lateral one to reduce hypoxia index, when any deceleration appears in labor.
As the hypoxia index covers the role of deceleration pattern classification, including early, late and variable decelerations, thus, FHR diagnosis is changing to quantitative logical ones from visual FHR deceleration pattern classification.

As Cahill et al. [13] also reported to predict UApH with the FHR deceleration area, novel quantitative logical diagnosis of the clinical outcome by fetal monitoring will change fetal monitoring to a computerized system instead of human visual analysis of FHR records.

\section{Conclusion}

As intrapartum FHR changes are complicated due to the mixture of FHR rise which is related fetal brain activity, and FHR fall which is related fetal hypoxia, thus, it is mandatory to study the roles of FHR rise and fall in fetal monitoring, notfuly rely on the FHR pattern classification, of which importance is changing according to the digital numeric analysis of FHR, where the rise and fall of FHR should be correctly analyzed according to their scientific facts. The author's hypoxia index and Cahill's deceleration area [13] are a good example of the update tendency of modern FHR diagnosis.

\section{Conflicts of Interest}

The authors declare that there is no conflict of interest regarding the publication of this paper. 


\section{References}

1. Hon EH (1968) An Atlas of Fetal Heart Rate Patterns. Harty Press, New Haven.

2. Hammacher K, Einfuehrung in die Cardiotokograph, 2. Die Schweizer Hebamme74 Jahrgang, Nr 6. 6-8.

3. Maeda K (2014) Modalities of fetal evaluation to detect fetal compromise prior to the development of significant neurological damage. J Obstet Gynaecol Res 40(10): pp. 2089-2094.

4. Maeda K (2016) Actocardiogram: Analysis of Fetal Motion and Heart Rate. Jaypee, New Delhi.

5. Maeda K, Noguchi Y, Matsumoto F, et al. (2006) Quantitative fetal heart rate evaluation without pattern classification: FHR score and artificial neural network analysis. In Kurjak, Chervenak Eds. Textbook of Perinatal Medicine, 2nd edition London, Informa. pp. 1487-1495.

6. Maeda K, Kimura S, Nakano H, et al. (1969) Pathophysiology of Fetus, Fukuoka Printing, Fukuoka.

7. Maeda K (2013) Actocardiographic analysis of fetal hypoxia detected by the bradycardia, loss of fetal heart rate acceleration and long term variability. J Health Med Inform 4(1): pp. 118-122.
8. Terao T, Kawashima $Y$, Noto $H$, et al. (1984) Neurological control of fetal heart rate in 20 cases of anencephalic fetuses. Am J Obstet Gynecol 149(2): pp. 201-208.

9. Ito T, Maeda K, Takahashi H, et al. (1994) Differentiation between physiologic and pathologic sinusoidal FHR pattern by fetal actocardiogram. J Perinat Med 22(1): pp. 39-48.

10. Umezawa J (1975) Studies on the relation between heart rate and $\mathrm{PaO} 2$ in hypoxic rabbit: a comparative study for fetal heart rate change in labor. Acta Obstet Gynecol Jpn 28: pp. 1203-1212.

11. Caldeyro-Barcia R, Poseiro JJ, Mendez-Bauer C, et al. (1967) Effects of abnormal uterine contraction on fetal heat rate during labor. Proc 5th World Conf Gynecol Obst, Sydney.

12. Poseiro JJ, Mendex-Bauer SV, Caldeyro-Barcia R (1969) Effect of uterine contractions on maternal blood flow through the placenta. 8th PAHO Advisary Committee on Medical Research pp. 161-171.

13. Cahill AG, Tuuli MD, Stout MJ, et al. (2018) A prospective cohort study of fetal heart rate monitoring: deceleration area is predictive of fetal acidemia. Am J Obstet Gynecol 218(5): 523. e1-523.e12. 